\title{
FACTORS AFFECTING THE SUSTAINED USE OF CHATBOTS: AN ORGANIZATIONAL PERSPECTIVE
}

\author{
Karine Aoun Barakat and Amal Dabbous \\ Saint Joseph University of Beirut, Lebanon
}

\begin{abstract}
Artificial intelligence innovations including conversational user interfaces have become widely adopted within the banking sector, as they present numerous advantages such as cost reduction, increased efficiency and quicker servicing of customers. However, these benefits will materialize only if organizations ensure the sustained use of these technologies. Hence, identifying the main factors that drive the sustained use of these services is critical to assess new technology adoption given that a very high number of IT implementations might fail. Researchers have relied on technology acceptance models to analyze the adoption of these new technologies within organizations, however little is known about the factors that ensure their sustained use. Using a qualitative methodology based on in-depth interviews with employees of a leading bank operating in developing economies, the study addresses this shortcoming by investigating the factors that contribute to the sustained use of conversational user interfaces, taking chatbots as a case study. Findings reveal that eight main internal and external factors are essential to promote the sustained use of chatbots, the most prominent of which are: a positive human-technology relationship, a favorable corporate culture, and the technology's high perceived efficiency.
\end{abstract}

\section{KEYWORDS}

Technology Sustained Use, Artificial Intelligence, Chatbots, Organizational Perspective

\section{INTRODUCTION}

Artificial intelligence (AI) and the use of digital technologies including chatbots are shaping the banking industry and facilitating the transition from traditional to conversational channels. They represent the current market trend and are expected to be used within several segments of large companies (Wilson et al., 2017). According to a survey conducted by Oracle, $80 \%$ of the decision makers participating in a global study confirm that they already used or plan to use chatbots by 2020 (Business Insider, 2016).

The use of chatbots presents numerous advantages for banks and financial institutions. These benefits include cost reduction, increased efficiency and quicker servicing of customers. Embracing conversational user interfaces might therefore enrich the performance of an organization as this channel can offer advanced customer care and personalized services while saving time and costs. However, significant cost reduction and increased efficiency due to the adoption of conversational interfaces can only materialize if this technology is continuously used.

The concept of sustained use is different from first time acceptance as instead of investigating the initial use behavior of a new technology, it has a long-term focus and analyzes a person's or organization's use behavior and interaction with this system over a longer period of time (Bhattacherjee, 2001).

Previous studies in the literature have carried research and developed theories that explain the acceptance and adoption of a new technology such as Venkatesh et al. (2003). However, none of these studies investigate the factors that drive the sustained use of a new technology. Although initial acceptance and use of new technologies can depict its success rate at the beginning, an information technology's long-term success rate and viability largely depends on its sustained and continuous use (Ratten, 2016; Wang et al., 2008). Continuous use behavior is therefore the extension of the initial use and is considered a critical factor to assess when adopting a new technology, particularly that some researchers advocate that almost $70 \%$ of all IT implementations fail (Balogun, \& Hope Hailey, 2004). Hence it is crucial to depict and specify which factors foster the sustained use of a new technology within an organization. 
This study aims to determine the significant factors that affect the sustained use of chatbots within an organization. To answer the research question, the study adopts an organizational perspective to conduct a qualitative analysis. Data collected from semi-structured interviews conducted with employees from a leading bank operating in several developing economies is examined using thematic analysis. Findings identify eight internal and external factors that promote the sustained use of conversational user interfaces.

To the best of the authors' knowledge, this study is considered among the first to examine factors that influence the sustained of use of chatbots within the banking environment context and to classify these factors as internal and external drivers for the continuous use of a new technology. Moreover, the findings of this study constitute the building block to develop and test a conceptual model for the sustained use of conversational user interfaces.

The paper is organized as follows, section two presents a summary of the concepts of Artificial Intelligence and chatbots and a review of the literature on technology acceptance, use and sustained use, section three develops the research methodology, section four reports and discusses the findings and section five concludes and portrays contributions and future research directions.

\section{THEORETICAL BACKGROUND}

\subsection{Artificial Intelligence and Chatbots}

Numerous financial institutions including banks are beginning to introduce within their activities various types of AI technologies namely those based on voice and text such as chatbots. A conversational interface or natural user interface is defined as an interface that the user can interact with by using natural inputs such as, speech, touch or hand gestures (Wigdor \& Wixon, 2011). This study focuses only on chatbots that use textual inputs.

These conversational AIs present the advantage of combining historical interactions with current real time data as well as internal and external data. Hence, this process makes the individualized engagement by a machine or a robot possible through the use of big data. Most importantly, this technology offers the advantages of reducing costs while making organizational processes more efficient which in turn enhances the value to both organizations and customers (Pannu, 2015).

Several factors encourage the adoption of chatbots by banks, the most important factor being the reduced cost of data storage, analytical tools and the advancements in machine learning which permit the automation of repetitive customer support tasks and will eventually allow the processing of more complicated interactions at lower costs. Furthermore, as more firms and consumers use chatbots, the increased demand for this technology will trigger additional development of chatbots, making the implementation process easier for organizations. Moreover, chatbot features are improving, thus making mass personalization possible due to innovations in artificial intelligence and machine learning technologies, big data analysis, improved analytics as well as predictive models. As a result, the number of financial institutions using chatbots and consumers that adopt this channel of communication will increase as the quality of delivered services improves across industries. Even though chatbots can be used in several segments such as marketing and payments, they are most frequently used for servicing customers and providing information to them. Within the global market, almost $45 \%$ of end users favor the use of chatbots as the primary channel of communication for customer service queries (Nguyen, 2017).

While the use of chatbots presents various advantages for banks and financial institutions, in order for these organizations to leverage this inexpensive and wide-reaching channel of communication to engage with a larger number of customers, it is important to depict the factors that are essential to ensure the sustained use of this technology within the organization. Identifying these factors is of major importance given that the use of this conversational user interface can face many challenges both internally and externally.

\subsection{Technology Acceptance, Implementation and Use}

\subsubsection{Theoretical Models}

Previous studies in the literature discussed the concept of technology acceptance and adoption. These studies primarily focused on the issues of technology acceptance explaining the technical aspects of design and 
implementation in a particular ICT infrastructure; among others we cite, Kane (2016) and Dahiya, (2017). Theories such as the technology acceptance model, TAM (Venkatesh \& Davis, 2000), the unified theory of acceptance and use of technology, UTAUT (Venkatesh et al., 2003) have been widely used to investigate the intention to adopt and use a new technology. These theories are nevertheless primarily concerned with the initial adoption of a technology and not its continuous and sustained use. They have been criticized for focusing on the exploitation stage and trying to forecast and model the behavior of the users that take the decision to adopt the technology or reject it (Aizstrauta et al., 2015).

Studies that proceed to further analyze the factors that ensure the sustained use of a technology are important because interiorized usage cannot be predicted by studies based solely on concepts such as acceptance and adoption or short-term use (Al-Sharafi et al., 2017). Sustained use also called continued use by some authors (Jennex \& Olfman, 2006) has a long term focus and analyzes the use behaviour and interaction with a particular system over a long period of time (Bhattacherjee, 2001). A given technology is not considered successful unless its usage is sustained by users who are supposed to benefit from this use (Bhattacherjee \& Lin, 2015). However, the literature tackling this issue is very scarce and to the best of the authors' knowledge none of the previous studies explored the factors that affect the sustained use of chatbots within an organization.

To further investigate the sustained use of a new technology within the existing literature, the IASAM2 of Aizstrauta et al. (2015) provides an in-depth view of a new technology sustainability and proposes to calculate an index to measure it. According to IASAM2 model, technology sustainability depends on four flows: Management, Quality of Technology, Acceptance and Domain Development. Management of the technology development and the quality of the technology used are considered to be internal flows as they are controlled by the organization itself, while technology acceptance and domain development are considered as external flows. Each of these flows is characterized by various socio-technical factors. However, the IASAM2 model presents a very large number of factors that affect the sustainability of a technology and is more suitable for a quantitative study. As the scope of the present study is to identify the most important factors that support the sustained use of a technology within an organization, this model is not suitable and an exploratory approach is used with the aim of refining the conceptual understanding of the sustained phenomenon under investigation.

\subsubsection{Sustained Use}

To explore the sustained use behaviour, this study draws on the technology adoption and use literature to identify possible internal and external factors that might influence the sustained use of a new technology. Particularly, among the internal factors that could affect the adoption of a new technology is its expected ability to fulfill the organization's needs (Kim et al., 2013) and to improve the job performance (Venkatesh et al., 2003). In addition, some advocate that the use of a new technology such as the conversational interface could replace human-based customer service capabilities, hence threatening the job security of the organization's employees. It is known that the use of a new technology within the organization will modify several organizational processes and tasks, hence affecting the nature of jobs (Dewett \& Jones, 2001; Taylor, 2004). Findings from the literature reveal that the success of the implementation of a new technology and the use of this technology by employees depends on their perceptions of how this new technology might affect their jobs (Bhattacherjee \& Premjumar, 2004; Owen \& Demb, 2004). Employees who perceive that the new technology will enhance their ability to accomplish their job tasks are more willing to adopt and use this new technology and might help to strengthen the technology adoption-performance link (Schraeder et al., 2006). Inversely, those who feel threatened by this new technology and are unwilling to embrace change will hinder the implantation and use of the technology.

Moreover, previous studies identify organizational culture as one of the factors that have a large influence on innovation within an organization as it could both stimulate or restrain the development and use of this innovation (Naranjo-Valencia et al., 2016). Organizational culture affects employees' behaviour and can lead the staff to consider innovation as a fundamental value of the organization and to feel more engaged in it (Hartmann, 2006). Stakeholders and leaders have also been considered to play an important role to facilitate the transition phase and transfer people from one state to another (Elrod \& Tippett, 2002), which might drive the sustained use of a new technology. Finally, as the degree to which a technology is used is determined by the sum of its use by individual users, another factor that influences the transition to conversational engagement and sustained use of this channel is the consumers' acceptance for this conversational interface. Hence, it is fundamental to continuously adjust, change and modify the processes within the organization when using a new technology to meet the expectations and the needs of the users (Bouwman et al., 2005). 
Several external factors could also affect the adoption of a new technology, some of which are directly linked to the industry or market regulations (Bouwman et al., 2005). In fact, the adoption of a new technology would be promoted under the existence of a favorable macro-policy, detailed regulations and rules that do not hinder the development of this technology (Shen et al., 2015). The way a technological product is used is also highly affected by the technical and social contexts. The technical context could restrain what the technology can achieve, while the social aspect establishes what will be acceptable in a given social system (Liebenau \& Harindranath, 2002). In addition, the decision to adopt a new technology could be either specific to the individuals or organizations that chose to adopt this technology or associated with environmental factors and characteristics specific to this innovation (Rogers, 2003; Frambach \& Schillewaert, 2002).

\section{METHODOLOGY}

The aim of this research being to understand the factors that lead to the sustained use of technology, a qualitative research approach was chosen. Qualitative studies capture the reality in substantial detail and are particularly useful when focusing on contemporary subjects (Chan and Ngai, 2007).

For this purpose, ten face-to-face semi-structured interviews with managers and employees within a bank that has implemented and is currently using a chatbot were conducted. The bank which is the object of this study operates in several developing economies and is considered to have a high level of experience with the chatbot as it has been continuously used for over a year and currently carries out 3,500 successful conversations every month. Interviewees were selected based on their experience as decision makers, implementers and users of the chatbot within the bank. Five of the employees which were interviewed are involved in the project as managers and strategic decision makers and the other five are implicated at the operation level and have a daily contact with the chatbot.

The guiding conceptual framework used during the interviews revolved around understanding the interaction between the organization itself, employees, the technology and the environment (Linton, 2002). The questions included in the interview guide aimed to determine the socio-technical factors that impacted the way the chatbot technology was developed, implemented and maintained, and the way in which this contributed to the continuous use of this technology.

Data collected through the ten interviews was then thematically analyzed. Thematic analysis can be widely used across a range of epistemologies and research questions. It is a method for identifying, analyzing organizing, describing and reporting themes found within a data set (Braun and Clarke, 2006). Within the context of this paper, a six-step analysis approach was adopted. Firstly, all the collected data was carefully read, and the sections which are of interest to the research were identified and captured. Secondly, initial codes were manually generated from the data by the two authors. Thirdly, the coded data extracts were sorted and collated into potential themes using an inductive approach. During the fourth step, themes were refined; those with not enough data to support them were merged into other themes and others which were too broad were broken down into separate themes. During the fifth step, the authors determined the aspects of the data each theme captured. The final step consisted of analyzing each theme and transcribing the findings (Braun and Clarke, 2006, Nowell et al, 2017).

\section{FINDINGS AND DISCUSSION}

Following the collection, coding, collation, refinement and analysis of the data derived from the ten face-to-face interviews, the resulting themes along with their corresponding verbatims were reported in Table 1 below. Some themes which were not adequately supported were excluded or integrated into other themes such as: the contribution to employees' job enhancement, top management support, and having realistic expectations about the technology. 
Table 1. Thematic analysis results

\begin{tabular}{|c|c|}
\hline Themes & Verbatim \\
\hline $\begin{array}{l}\text { Favorable Corporate } \\
\text { Culture }\end{array}$ & $\begin{array}{l}\text { "The bank already has a mindset of continuous growth... The technology culture } \\
\text { is there and has always been there" } \\
\text { "The culture change that now accepts failure has led us to unleash our innovation } \\
\text { capabilities" } \\
\text { "We are a bank which loves to innovate and try new things...it's a question of } \\
\text { having the right mentality geared towards openness and new technologies" }\end{array}$ \\
\hline $\begin{array}{l}\text { Technological } \\
\text { Advancement }\end{array}$ & $\begin{array}{l}\text { "There is a general trend towards using chatbots around the world" } \\
\text { "Once the chatbot technology became accessible to end users we thought it was } \\
\text { time to start using it" } \\
\text { "The chatbot can grow as far as technology allows it" }\end{array}$ \\
\hline Perceived Efficiency & $\begin{array}{l}\text { "The chatbot enhances both the efficiency and the customer experience at the } \\
\text { bank" } \\
\text { "Chatbot use has increased customer satisfaction" } \\
\text { "The chatbot allows us to save time" }\end{array}$ \\
\hline $\begin{array}{l}\text { Compliance } \\
\text { Regulations }\end{array}$ & $\begin{array}{l}\text { "The scope of the chatbot is being hindered because of the regulatory framework" } \\
\text { "Regulations remain however a hurdle to developing chatbots further especially } \\
\text { that they can sometimes be difficult to interpret" }\end{array}$ \\
\hline $\begin{array}{l}\text { Positive Human- } \\
\text { Technology Relationship }\end{array}$ & $\begin{array}{l}\text { "No jobs were lost, no threats... On the contrary new jobs were created" } \\
\text { "Our chatbot was given an identity, a name...employees working with the chatbot } \\
\text { consider it as their baby" } \\
\text { "We are very happy to have the chatbot, we love him a lot" } \\
\text { "Customers have easily accepted the idea of chatting with a bot" } \\
\text { "More and more customers prefer to interact with the chatbot than contact us } \\
\text { through our call center" }\end{array}$ \\
\hline $\begin{array}{l}\text { Multi-Stakeholder } \\
\text { Implication }\end{array}$ & $\begin{array}{l}\text { "All parties are on-board" } \\
\text { "People from different departments are constantly solicited to be involved in the } \\
\text { project" } \\
\text { "Our vendors have to understand how we work, they have to be part of the team" }\end{array}$ \\
\hline Continuous Improvement & $\begin{array}{l}\text { "We have a roadmap of enhancements...we are continually working on } \\
\text { improving the chatbot" } \\
\text { "Technology enhancements cannot only be done from the company side, we need } \\
\text { to know what customers require" } \\
\text { "Continuous development not only from the operational side but at the managerial } \\
\text { and strategic level as well is essential" }\end{array}$ \\
\hline Strategic Alignment & $\begin{array}{l}\text { "In line with the bank's positioning as a leader in the field of technology" } \\
\text { "Our customers and competitors admire the Bank's innovations" } \\
\text { "Having a chatbot reflects positively on the bank's image as a pioneer and } \\
\text { innovator" }\end{array}$ \\
\hline
\end{tabular}

After reviewing the resulting eight themes, a distinction appeared between factors that could be controlled by the bank and those that are the product of its environment. Results are summarized in Figure 1 below. For instance, when discussing the technological advancement, it appeared clearly that the bank was dependent on the evolution of the AI technology environment as a whole and that in this case the sustained and continuous use of the chatbot was tied to how fast and far this technology would progress. Likewise, the prolonged use of the chatbot relies to a great extent on the regulatory environment and can be hindered in case stricter laws are passed. In terms of factors that could be controlled by the company which are favorable to the continuous use of chatbots, these were: having a corporate culture that encourages innovation, the technology's perceived efficiency, the multi-stakeholder approach mentality, the will to continuously improve and enhance the technology, as well as the alignment of the technology with the company's strategy. The final key factor which appeared was a positive human-technology relationship where not only employees and technology work side by side, but also customers have readily accepted and are satisfied with using this technology. 


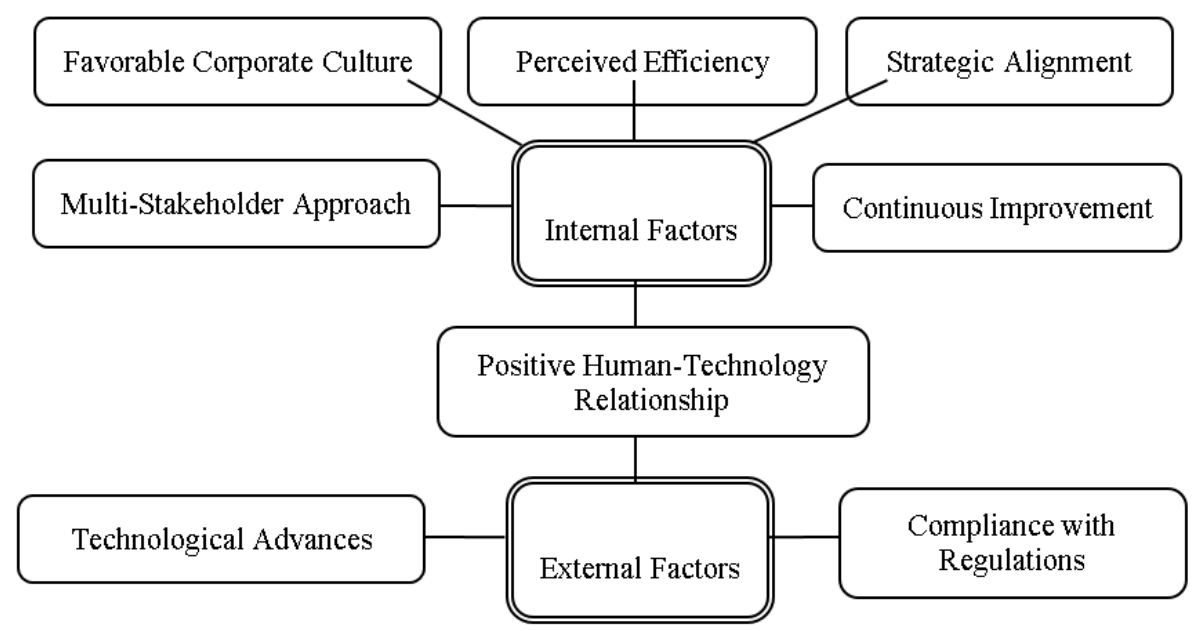

Figure 1. Factors affecting the continuous use of chatbots

Furthermore, among the noteworthy findings of the study was identifying which of the eight factors initially appear during the adoption phase of the technology and continue to play a crucial role in maintaining the continuous use of this technology. The first of these factors is strategic alignment, as mentioned by Lin \& Chen (2012), who considered that a key factor affecting cloud computing adoption is the technology's compatibility with the company's business model and customer demands. Another such factor is the perceived efficiency, which proved to be a motivator for travel and tourism companies adopting robots as it translates into a reduction of costs and an increase in productivity (Ivanov \& Webster, 2017). Finally, the technology's compliance with regulations is considered as a vital factor which determines its adoption, given that any new technology would be promoted under the existence of a favorable macro-policy, as well as detailed regulations and rules that do not hinder its development (Shen et al., 2015).

\section{CONCLUSION}

The present study contributes to the literature on the use of technology by including the sustained use behavior and identifying eight main internal and external factors that are considered essential to ensure the sustained use of chatbots within an organization. Semi structured interviews were conducted and a thematic analysis was adopted to select and validate eight main themes which are considered crucial internal and external factors that affect the sustained use of conversational user interfaces like chatbots.

The findings can be used both by academics and practitioners. For academics, it enriches the current models by including a long-term view into the use of technology, particularly chatbots. And as such, it opens new research streams. For practitioners, the paper which is based on a case study highlights the essential factors managers should take into account to ensure sustained use when adopting a new technology. Identifying these factors is crucial as it will help decision makers within an organization to increase proficiency, decide on the suitability of a new technology adoption and whether this technology can be continuously used or not. It stems from the authors' belief that acceptance and adoption are not enough to justify the substantial investments made by companies in the field of technology.

Even though the organization chosen to conduct this study operates in different developing countries, the findings of this research will have the typical limitations and threats to validity of a case study research, particularly with respect to generalization of results to other regions. Other limitations relate to the sector of activity of the studied organization which might also make the generalization of results difficult for organizations which are not in the banking sector.

As a future work, it would be interesting to include a larger number of organizations operating in different geographical regions and in different sectors of activity to increase the validity and generalizability of the results. Moreover, the next step could entail an in-depth quantitative analysis to hypothesize on the relations between the identified factors and their impact on the sustained use of chatbots within organizations. These hypotheses will constitute the basis for a new testable research model. 


\section{REFERENCES}

Aizstrauta, D., et al., 2015. Applying theory of diffusion of innovations to evaluate technology acceptance and sustainability. Procedia Computer Science, 43, pp. 69-77.

Al-Sharafi, M.A., et al., 2017. Factors Influencing the Continuous Use of Cloud Computing Services in Organization Level. In Proceedings of the International Conference on Advances in Image Processing (pp. 189-194). ACM.

Balogun, J. and Hope Hailey, V., 2004. Exploring Strategic Change. Prentice Hall, London.

Bhattacherjee, A., 2001. Understanding Information Systems Continuance: An Expectation-Confirmation Model. MIS Quarterly, 25 (3), pp. 351-370.

Bhattacherjee, A., and Lin, C. P., 2015. A unified model of IT continuance: three complementary perspectives and crossover effects. European Journal of Information Systems, 24(4), pp. 364-373.

Bhattacherjee, A., and Premjumar, G., 2004. Understanding changes in belief and attitude toward information technology usage: A theoretical model and longitudinal test. MIS Quarterly, 28(2), pp. 229-254.

Bouwman, H., et al., 2005. Information and communication technology in organizations: adoption, implementation, use and effects, Sage.

Braun, V. and Clarke, V., 2006. Using thematic analysis in psychology. Qualitative research in psychology, 3(2), pp 77-101.

Chan, S. C. and Ngai, E. W., 2007. A qualitative study of information technology adoption: how ten organizations adopted Web-based training. Information Systems Journal, 17(3), pp. 289-315.

Dahiya, M., 2017. A Tool of Conversation: Chatbot. International Journal of Computer Sciences and Engineering, 5(5), pp. 158-161.

Dewett, T., and Jones, G. R., 2001. The role of information technology in the organization: A review, model, and assessment. Journal of Management, 27, pp. 313-346.

Elrod, P. D., and Tippett, D. D., 2002. The "Death Valley" of change. Journal of organizational change management, 15(3), pp. 273-291.

Frambach, R. T., and Schillewaert, N., 2002. Organizational innovation adoption: A multilevel framework of determinants and opportunities for future research. Journal of Business Research, 55(2), pp.163-176.

Hartmann, A., 2006. The role of organizational culture in motivating innovative behaviour in construction firms. Construction Innovation, 6(3), pp. 159-172.

Ivanov, S. H., \& Webster, C., 2017. Adoption of robots, artificial intelligence and service automation by travel, tourism and hospitality companies-a cost-benefit analysis. Artificial Intelligence and Service Automation by Travel, Tourism and Hospitality Companies-A Cost-Benefit Analysis.

Jennex, M.E., and Olfman, L., 2006. A Model of Knowledge Management Success. International Journal of Knowledge Management, vol. 2, pp. 51-68.

Kane, D. A., 2016. The Role of Chatbots in Teaching and Learning. E-Learning and the Academic Library: Essays on Innovative Initiatives, 131.

Kim, Y. H., et al., 2013. A study of mobile user engagement (MoEN): Engagement motivations, perceived value, satisfaction, and continued engagement intention. Decision Support Systems, 56, pp. 361-370.

Liebenau, J. and Harindranath, G., 2002. Organizational reconciliation and its implications for organizational decision support systems: a semiotic approach. Decision Support Systems, 33(4): pp. 339-398.

Lin, A., \& Chen, N. C., 2012. Cloud computing as an innovation: Percepetion, attitude, and adoption. International Journal of Information Management, 32(6), 533-540.

Linton, J.D., 2002. Implementation research: state of the art and future directions, Technovation, 22(2), pp. 65- 79.

Naranjo-Valencia, J. C., et al., 2016. Studying the links between organizational culture, innovation, and performance in Spanish companies. Revista Latinoamericana de Psicología, 48(1), pp. 30-41.

Nguyen, M.H., 2017. The latest market research, trends \& landscape in the growing AI chatbot industry. Business Insider, October issue.

Nowell, L. S., et al., 2017. Thematic analysis: Striving to meet the trustworthiness criteria. International Journal of Qualitative Methods, 16(1), 1609406917733847.

Owen, P. S. and Demb, A., 2004. Change dynamics and leadership in technology implementation. Journal of Higher Education, 75(6), pp. 636-666.

Pannu, A., 2015. Artificial intelligence and its application in different areas. Artificial Intelligence, 4(10), pp. 79-84.

Ratten, V., 2016. Continuance use intention of cloud computing: Innovativeness and creativity perspectives, Journal of Business Research, 69(5), pp. 1737-1740.

Rogers E.M., 2003. Diffusion of Innovations. Free Press, New York. 
Schraeder, M., et al., 2006. Employee involvement, attitudes and reactions to technology changes. Journal of Leadership \& Organizational Studies, 12(3), 85.

Shen, G., et al., 2015. Factors influencing the adoption and sustainable use of clean fuels and cookstoves in China-a Chinese literature review. Renewable and Sustainable Energy Reviews, 51, pp.741-750.

Taylor, D. S., 2004. Technology acceptance: Increasing new technology use by applying the right messages. Performance Improvement, 43(9), pp. 21-26.

Trott, P., 2001. The Role of Market Research in the Development of Discontinuous New Products. European Journal of Innovation Management, 4, pp. 117-125

Venkatesh, V., and Davis, F. D., 2000. A theoretical extension of the technology acceptance model: Four longitudinal field studies. Management Science, 46(2), pp. 186-204.

Venkatesh, V., et al., 2003. User acceptance of information technology: Toward a unified view. MIS Quarterly, 27(3)

Wang, S.C., et al., 2008. A study on the continuous adoption intention model of information systems-cognitive process, emotional states, and belief bases. In Service Systems and Service Management International Conference

Wigdor, D., and Wixon, D., 2011. Brave NUI world: designing natural user interfaces for touch and gesture. Elsevier. Wilson, H. J., et al., 2017. The jobs that artificial intelligence will create. MIT Sloan Management Review, 58(4), 14-17

Business Insider, 2016 https://www.businessinsider.com/80-of-businesses-want-chatbots-by-2020-2016-12 